\title{
Time-dependent topological systems: A study of the Bott index
}

\author{
Daniele Toniolo* \\ Department Mathematik, Friedrich-Alexander-Universität Erlangen-Nürnberg, Erlangen, Germany
}

(Received 28 September 2018; published 21 December 2018)

\begin{abstract}
The Bott index is an index that discerns among pairs of unitary matrices that can or cannot be approximated by a pair of commuting unitary matrices. It has been successfully employed to describe the approximate integer quantization of the transverse conductance of a system described by a short-range, bounded, and spectrally gapped Hamiltonian on a lattice on a finite two-dimensional torus and to describe the invariant of the BernevigHughes-Zhang model even with disorder. This paper shows the constancy in time of the Bott index and the Chern number related to the time-evolved Fermi projection of a system described by a short-range, bounded, and time-dependent Hamiltonian that is initially gapped. The general situation of a ramp of a time-dependent perturbation is considered, a section is dedicated to time-periodic perturbations.
\end{abstract}

DOI: 10.1103/PhysRevB.98.235425

\section{INTRODUCTION}

A recent focus of the research in condensed matter has been the description of the topological properties of systems that are subjected to perturbations in particular to time-driven ones. Early works on this subject are Refs. [1-3]. These consider how a time-periodic drive can induce a topological phase in the context of the Bernevig-Hughes-Zhang model and for a two-dimensional system of fermions on a honeycomb lattice with spectral gap, respectively. More recent studies [4-9] have realized that the Chern number is invariant under the unitary time evolution of the system, moreover, in a two-dimensional setting when the Chern number of the initial ground state and that of the ground state of the instantaneous Hamiltonian are different, then the Hall conductance is no more quantized. This has been shown both for the case of a quench from an initial trivial state to a topological one in the Haldane model $[6,8]$ and in the case of a system of spinless fermions of a honeycomb lattice described by an initial gapped Hamiltonian that is subjected to the linear ramp of a periodic external electromagnetic field [5,7]. The topology of periodically driven systems has been intensively studied; an incomplete list of works includes the references that have introduced and rigorously discussed the $W$ invariant in two dimensions $[10,11]$ and in any dimension with the use of a $K$-theoretic construction [12], the study of the chiral case [13], and the study of the time-reversal-invariant case [14] (for the experimental side see [15] and references therein). The invariance of the topological properties of the ground states, in general degenerate, of gapped Hamiltonians with respect to local unitarity transformations has been studied by Hastings and Wen [16]. Related ideas recently brought an explicit formulation of the adiabatic theorem in the manybody context $[17,18]$. A different approach to the dynamics of topological systems is the study of the winding of the Pancharatnam phase developed by the authors of [19].

*daniele.toniolo@fau.de
The Bott index is an index of matrices that has been employed in the condensed matter realm by Loring and Hastings [20]. The index has been introduced in Ref. [21], and its mathematical and physical foundations have been studied in [22,23] (see also [24] and references therein). The Bott index discerns among a couple of unitary or almost unitary matrices that can or cannot be approximated by a commuting pair. In this paper, we will be concerned with the index of a couple of unitary or quasiunitary matrices, while the index has been used also for triples of Hermitian matrices and for other classes in the case of systems with symmetries, time reversal, or particle hole. Accordingly, a general classification of the topological phases of any of the Altland-Zirnbauer symmetry classes has been developed in [22,23]. The Bott index of the projected position matrices on a torus, as defined by Eq. (11) below, states whether those matrices can or cannot be approximated by a couple of commuting matrices. This encodes an information about the localization properties of the Fermi projection $P$. The existence of exponentially localized Wannier functions implies the vanishing of the Bott index; a vanishing Bott index implies a small variance of the Wannier functions with respect to the system's size [23]. Recently, the relation among the spread and the localization of Wannier functions has been investigated in [25]. The Bott index is well suited for numerical simulations being designed for finite systems; it also handles the effects of disorder.

The main subject of this work is to show that the Bott index of the time-evolved Fermi projection of a two-dimensional system described by a finite-ranged, bounded, and initially gapped Hamiltonian is constant in time in the thermodynamic limit when the Hamiltonian is subjected to a perturbation in general time dependent that preserves the locality of the Hamiltonian. A possible change of the index in a certain timescale is only a finite-size effect; this is the content of Eq. (25). The result is model independent within the stated hypothesis on the Hamiltonian. An analogous numerical result for a specific model limited to a time-periodic perturbation was provided in Ref. [26]. Another result of this work is to show that the Chern number of the time-evolved Fermi 
projection is constant in time under the same hypothesis for the Hamiltonian as in the Bott index case but in an infinite two-dimensional system. This also proves that the Bott index and the two-dimensional Chern number are equivalent both in a time-independent setting [23,26,27] and in a time-dependent one. A related result regarding the topological order of a set of degenerate ground states has been obtained in Ref. [28].

The structure of the paper is as follows: In Sec. II the invariance of the two-dimensional Chern number along the time evolution is proved. The Bott index is introduced in Sec. III and the requirements on the physical setting for the index to be well defined are described. The spectral flow that shows the mechanism of a possible variation of the Bott index is discussed in the unitary and in the general case. In Sec. IV the growth in time of the norm of the commutator among the time-evolved Fermi projection and the position operator is studied and it is shown that for finite-range Hamiltonians it cannot give rise to a change of the Bott index. Some technical details are in the Appendix. $\hbar=1$ throughout the paper.

\section{INVARIANCE OF THE TWO-DIMENSIONAL CHERN NUMBER OF THE TIME-EVOLVED FERMI PROJECTION}

Let us consider a two-dimensional insulating system of noninteracting particles with $N$ internal degrees of freedom on an infinite lattice, that for convenience we take equal to $\mathbb{Z}^{2}$, described by a single-particle gapped Hamiltonian $H$ of finite range $R$ acting on the Hilbert space $l^{2}\left(\mathbb{Z}^{2}\right) \otimes \mathbb{C}^{N}$ :

$$
H=\sum_{l, k=1}^{N} \sum_{\|n-m\| \leqslant R} \mathcal{H}_{n, m, l, k}|n, l\rangle\langle m, k| .
$$

The finite-range condition reads as follows: if $\|n-m\|>R$, then $\mathcal{H}_{n, m, l, k}=0$. $\left\{|n\rangle, n=\left(n_{x}, n_{y}\right) \in \mathbb{Z}^{2}\right\}$ is the usual basis of $l^{2}\left(\mathbb{Z}^{2}\right)$ such that $|n\rangle$ equals 1 at the site $n$ and zero elsewhere. The Fermi level $\mu$ is supposed to lie in an energy gap of $H$. The expression of the Chern number of the Fermi projection $P \equiv \chi(H \leqslant \mu)$, here defined by the functional calculus with $\chi$ the characteristic function, suitable for an evaluation of this topological invariant in real space has been employed, for example, as Eq. (19) of [29] and more recently in the Appendix C of Kitaev [30]. See also Eq. (7) of [31] and [32,33]:

$$
\operatorname{Chern}(P) \equiv 4 \pi \operatorname{Im} \operatorname{Tr}_{\text {u.a. }} Q[X, P] P[Y, P] .
$$

Note that there is no uniformity in the choice of the sign of the Chern number in the literature. $\operatorname{Tr}_{\text {u.a. }}$ is the trace per unit area: $\operatorname{Tr}_{\text {u.a. }} \equiv \lim _{A \rightarrow \infty} \frac{\operatorname{Tr} \chi_{A}}{A}, \chi_{A}$ denotes multiplication by the characteristic function of the area $A$, that is 1 inside $A$, zero outside. $Q$ is the projection orthogonal to $P, Q \equiv \mathbb{1}-P$. The operators $X$ and $Y$ in Eq. (1) are the position operators of $l^{2}\left(\mathbb{Z}^{2}\right)$. For a discussion of the convergence of the trace that defines the Chern number, see the so-called Sobolev condition described, for example, in [29,34]. For a reformulation of the Chern number using switch functions instead of position operators and the inclusion of a wider class of Hamiltonian with exponentially decreasing amplitudes see, for instance, $[11,35]$. Another perspective with equivalent results for the quantization of the Hall conductance is that of Avron et al.
[36] that considered the Fredholm index of an operator associated to a couple of projections.

The constancy in time of the Chern number when the unitary time evolution of the system is taken into account has been already shown analytically in [5] for a spatially periodic system following a time evolution where a periodic perturbation is turned on and in Ref. [6] through a numerical evidence for the Haldane model following a quench. In both cases, the Schrödinger picture for the unitary evolution was employed. This means that, in the present notation, the quantity

$$
\begin{aligned}
\operatorname{Chern}\left(P\left(t, t_{0}\right)\right)= & 4 \pi \operatorname{Im}_{\operatorname{Tr}_{\text {u.a. }} Q\left(t, t_{0}\right)\left[X, P\left(t, t_{0}\right)\right] P\left(t, t_{0}\right)} \\
& \times\left[Y, P\left(t, t_{0}\right)\right]
\end{aligned}
$$

has been studied, where $P\left(t, t_{0}\right) \equiv U\left(t, t_{0}\right) P\left(t_{0}\right) U^{\dagger}\left(t, t_{0}\right)$, and it has been shown to be independent of $t$ with a local Hamiltonian $H(t) . U\left(t, t_{0}\right)$ is the unitary operator of time evolution of the system satisfying

$$
i \partial_{t} U\left(t, t_{0}\right)=H(t) U\left(t, t_{0}\right), \quad U\left(t_{0}, t_{0}\right)=\mathbb{1} .
$$

For a time-independent system, the time evolution is given by $U\left(t, t_{0}\right)=e^{-i H\left(t-t_{0}\right)}$, then the invariance of the Chern number is manifest, therefore the relevant fact is the invariance in the general case of a system with a time-dependent Hamiltonian $H(t)$ [see Eq. (20)]. The conditions required for the invariance of the Chern number under unitary evolution according to Ref. [5] are the locality of the instantaneous Hamiltonian $H(t)$ in Eq. (3) and certain regularity properties of the ground-state projection over the Brillouin zone. In the present setting I consider the instantaneous Hamiltonian $H(t)$ finite range and in general gapped only at the initial time $t_{0}$; this ensures the regularity of the projections $P\left(t_{0}\right)$ and $Q\left(t_{0}\right)$ as discussed, for example, in [31]. I present an alternative proof of the constancy in time of the Chern number, $\operatorname{Chern}\left(P\left(t, t_{0}\right)\right)=$ Chern $\left(P\left(t_{0}\right)\right)$. To this purpose, the Chern number is expressed with the aid of switch functions $\Lambda_{x}$ and $\Lambda_{y}$ defined as follows: it exists a positive integer $M$ such that with $x>M, \Lambda_{x}(x)=$ 1 and with $x<-M, \Lambda_{x}(x)=0$ and $\Lambda_{x}$ varies smoothly in-between. $\Lambda_{y}$ is similarly defined. Using the functional calculus the operators $\Lambda_{x}(X)$ and $\Lambda_{y}(Y)$ are defined, namely, $\Lambda_{x}(X)\left|n_{x}, n_{y}\right\rangle=\Lambda_{x}\left(n_{x}\right)\left|n_{x}, n_{y}\right\rangle$. With abuse of notation I will write in the following $\Lambda_{x}$ and $\Lambda_{y}$ for the corresponding operators. According to, for example, $[29,36]$

$$
\operatorname{Chern}(P)=4 \pi \operatorname{Im} \operatorname{Tr} Q\left[\Lambda_{x}, P\right] P\left[\Lambda_{y}, P\right] .
$$

The $\operatorname{Tr}$ is over the Hilbert space $l^{2}\left(\mathbb{Z}^{2}\right)$. In what follows, $t_{0}=0, P \equiv P\left(t_{0}\right)$, and $P(t) \equiv P\left(t, t_{0}\right)$. We want to show the invariance of the Chern when $P$ is replaced with $P(t)$. The Chern number of a projection is well defined when the trace in Eq. (4) is finite; in this case the Chern number turns out to be an integer. Projections that have well-defined Chern number and that are homotopically equivalent have the same Chern number; for a proof of this statement in the context of Fredholm-index theory see, for example, [37]. $P$ and $P(t)$ are homotopically equivalent, therefore, the task is to show that the trace in Eq. (4) is finite when replacing $P$ with $P(t)$. In what follows, it is convenient to consider a more general class of Hamiltonians than the finite-range ones, namely, the class of Hamiltonians with off-diagonal elements falling exponentially fast: $|\langle n|H| m\rangle| \leqslant M e^{-v\|n-m\|}$; this class 
of operators is called local. Let us consider

$$
\operatorname{Chern}(P(t))=4 \pi \operatorname{Tr} Q(t)\left[\Lambda_{x}, P(t)\right] P(t)\left[\Lambda_{y}, P(t)\right] .
$$

Defining the Heisenberg picture $X_{H}(t) \equiv U^{\dagger}(t) X U(t)$, with the aid of functional calculus we have

$$
\Lambda_{x}\left(X_{H}(t)\right)=U^{\dagger}(t) \Lambda_{x}(X) U(t) .
$$

Therefore, dropping the time index of $U(t)$, we have

$$
\operatorname{Chern}(P(t))=4 \pi \operatorname{Im} \operatorname{Tr} Q\left[U^{\dagger} \Lambda_{x} U, P\right] P\left[U^{\dagger} \Lambda_{y} U, P\right] .
$$

The equation of motion for $X_{H}(t)$ is

$$
i \frac{d}{d t} X_{H}(t)=[X, H(t)]_{H},
$$

where the explicit time dependence of the Hamiltonian has been put in evidence. With $X=X\left(t_{0}\right)$ we have

$$
i\left[X_{H}(t)-X\right]=\int_{0}^{t} d s[X, H(s)]_{H} .
$$

A simple manipulation of Eq. (5) leads to

$$
\begin{aligned}
\operatorname{Chern}(P(t))= & 4 \pi \operatorname{Im} \operatorname{Tr} Q\left[\left(\Lambda_{x}+U^{\dagger}\left[\Lambda_{x}, U\right]\right), P\right] \\
& \times P\left[\left(\Lambda_{y}+U^{\dagger}\left[\Lambda_{y}, U\right]\right), P\right] .
\end{aligned}
$$

We replace in the equation above

$$
U^{\dagger}\left[\Lambda_{x}, U\right]=-i \int_{0}^{t} d s U^{\dagger}(s)\left[\Lambda_{x}, H(s)\right] U(s)
$$

and similarly for the $y$ factor. We note that the operator $\left[\Lambda_{x}, H(s)\right]$ is confined around the $y$ axis; this follows from the definition of $\Lambda_{x}$ and from the locality of $H(s)$ with $s \in[0, t]$; this is discussed, for example, in Refs. [11,36]. We will denote this behavior saying that $\left[\Lambda_{x}, H(s)\right]$ is $x$ confined, namely, that it exists a positive constant $a$ such that the operator $\left[\Lambda_{x}, H(s)\right] e^{a|x|}$ is bounded (see Lemma 4.4 of Ref. [11]). In the case of a finite range $H(s)$ the $x$ confinement of $\left[\Lambda_{x}, H(s)\right]$ is easily understood.

To prove that the trace in Eq. (6) is finite and well defined, namely, basis independent, we need to show that the operator that we are tracing out is trace class (see [37] for the definition); to do so we show that it splits up as a sum of trace class operators, denoted $I, I I, I I I$, and $I V$.

$$
I \equiv Q\left[\Lambda_{x}, P\right] P\left[\Lambda_{y}, P\right]
$$

is trace class by hypothesis; this follows from the fact that the Fermi projection $P$ of a gapped and short-range, or local, Hamiltonian is local, namely, it has off-diagonal components falling off exponentially fast: $|\langle n|P| m\rangle| \leqslant K e^{-\mu\|n-m\|}$.

$$
I I \equiv-i \int_{0}^{t} d s Q\left[\Lambda_{x}, P\right] P U^{\dagger}(s)\left[\Lambda_{y}, H(s)\right] U(s)
$$

is also trace class, in fact, $\left[\Lambda_{x}, P\right]$ and $\left[\Lambda_{y}, H(s)\right]$ are, respectively, $x$ and $y$ confined, therefore, their product is trace class; moreover, since the product of a trace class operator and a bounded operator is trace class, it follows that $P U^{\dagger}\left[\Lambda_{y}, H(s)\right]\left[\Lambda_{x}, P\right]$ is trace class. Using the cyclic property of the trace, it follows that $\left[\Lambda_{x}, P\right] P U^{\dagger}\left[\Lambda_{y}, H(s)\right]$ is trace class. Using again the fact that the trace class operators are an ideal of the bounded operators, we get that (8) is trace class.

$$
\begin{aligned}
I I I \equiv & -i \int_{0}^{t} d s^{\prime} Q\left[\Lambda_{y}, P\right] P U^{\dagger}\left(s^{\prime}\right)\left[\Lambda_{x}, H\left(s^{\prime}\right)\right] U\left(s^{\prime}\right) \\
I V \equiv & \int_{0}^{t} d s d s^{\prime} Q U^{\dagger}(s)\left[\Lambda_{x}, H(s)\right] U(s) P U^{\dagger}\left(s^{\prime}\right) \\
& \times\left[\Lambda_{y}, H\left(s^{\prime}\right)\right] U\left(s^{\prime}\right) .
\end{aligned}
$$

Applying a similar reasoning as for $I I$ to $I I I$ and $I V$ we obtain that both are trace class.

The above trace class discussion together with the homotopy equivalence of $P$ and $P(t)$ shows that the value of the Chern number of $P(t)$ is constant in time under the hypothesis that the Hamiltonian of the system $H(s)$ is spatially local with $s \in[0, t]$. This also means that the sum of the contributions to the trace of the operators $I I, I I I$, and $I V$ is zero.

We note that in the reasoning above we have exchanged the time integration and the trace; this can be justified simply. We suppose that the time dependence of the Hamiltonian is at least strongly continuous; this ensures the existence of a dynamics for the system, namely, the propagator $U\left(t, t_{0}\right)$ of Eq. (3) is well defined (see paragraph X.12 of [38] for a discussion). We can approximate the time integration by a finite sum plus a small remainder. We can safely exchange the trace and the finite sum, moreover, since we conclude that the contribution of the finite sum is independent from $t$, that means the contribution of the remainder is vanishing.

\section{RECOLLECTING THE DEFINITION OF THE BOTT INDEX AND ITS RELATION WITH THE TWO-DIMENSIONAL CHERN NUMBER}

In Refs. [23,26,27] it has been shown that for a manybody system of noninteracting particles described by a shortranged, bounded, and gapped Hamiltonian living on a lattice on a finite two-torus, the invariant of matrices called Bott index coincides in the thermodynamic limit with the Chern number. In finite systems the correction is of order $L^{-1}$, being $L$ the linear size of the system. This in particular implies the quantization of the Hall conductance on a torus of finite size with an error of order $L^{-1}$. This has been shown in Ref. [23] exploiting the definition of the Hall conductance as the long time transverse current response of the system to an electric field adiabatically turned. The proof of Ref. [27] relies instead on a direct comparison of the invariants as they appear in Eqs. (1) and (18).

Following Ref. [22], we consider a representation of the position operators $X$ and $Y$ such that the positions of all the particles of the system $\left(x_{i}, y_{i}\right)$ are disposed on the diagonal of $X$ and $Y$, respectively: $X_{i, j}=x_{i} \delta_{i, j}$. Being $L$ the linear size of the system and assuming a lattice spacing equal to $1, X$ and $Y$ are matrices of size $N L^{2} \times N L^{2}$. Denoting as in Sec. II the Fermi projection with $P$,

$$
P=W\left(\begin{array}{cc}
0 & 0 \\
0 & \mathbb{1}_{n}
\end{array}\right) W^{\dagger}
$$


with $n=\operatorname{dim} P$ and $W$ the unitary matrix of basis change from energy to position, so we also have

$$
Q \equiv \mathbb{1}-P=W\left(\begin{array}{cc}
\mathbb{1}_{m} & 0 \\
0 & 0
\end{array}\right) W^{\dagger} .
$$

In a system with periodic boundary conditions, we consider the unitary matrices $e^{\left(i \frac{2 \pi X}{L}\right)}$ and $e^{\left(i \frac{2 \pi Y}{L}\right)}$. Then, for the projected position operators on a torus $P e^{\left(i \frac{2 \pi X}{L}\right)} P$ and $P e^{\left(i \frac{2 \pi Y}{L}\right)} P$, we have

$$
\begin{aligned}
& P e^{\left(i \frac{2 \pi X}{L}\right)} P=W\left(\begin{array}{cc}
0 & 0 \\
0 & U_{1}
\end{array}\right) W^{\dagger}, \\
& P e^{\left(i \frac{2 \pi Y}{L}\right)} P=W\left(\begin{array}{cc}
0 & 0 \\
0 & U_{2}
\end{array}\right) W^{\dagger},
\end{aligned}
$$

where $U_{1}$ and $U_{2}$ are nonsingular. With $R \ll L$ the range of the Hamiltonian, $J$ a bound for the norm of $H$, and $\Delta E$ the spectral gap of the Hamiltonian, it turns out $[22,23]$ that

$$
\begin{gathered}
\|[X, H]\| \leqslant O(R J), \\
\left\|\left[e^{\left(i \frac{2 \pi X}{L}\right)}, P\right]\right\| \leqslant O\left(\frac{R J}{L \Delta E}\right) .
\end{gathered}
$$

A couple of words on these relations: Eq. (13) follows from the fact that $X$ is a diagonal matrix, then $[X, H]$ looks like the off-diagonal part of $H$ with elements multiplied by factors of modulus at most equal to $R$ because $H$ connects lattice points that are at most $R$ far apart. A similar bound occurs in the case of local Hamiltonian on $l^{2}\left(\mathbb{Z}^{2}\right)$ where $R \propto \frac{1}{v}$. Equation (14) follows from (13) writing $P$ as a contour integral of the resolvent $R(z) \equiv(z-H)^{-1}$ (see [22] and [27] for more details). This implies that the matrices $U_{1}$ and $U_{2}$ almost commute and are quasiunitary:

$$
\begin{gathered}
\left\|\left[U_{1}, U_{2}\right]\right\| \leqslant O\left(\frac{R J}{L \Delta E}\right)^{2} \\
\left\|U_{a} U_{a}^{\dagger}-\mathbb{1}_{n}\right\| \leqslant O\left(\frac{R J}{L \Delta E}\right)^{2}, \quad a \in\{1,2\} .
\end{gathered}
$$

For a discussion of these results, see [23]. The Bott index of $U_{1}$ and $U_{2}$ is defined, according to [20], as

$$
\operatorname{Bott}\left(U_{1}, U_{2}\right) \equiv \frac{1}{2 \pi} \operatorname{Im} \operatorname{Tr} \log \left(U_{1} U_{2} U_{1}^{\dagger} U_{2}^{\dagger}\right) .
$$

The branch cut of the logarithm is assumed on the real negative axis, then the definition is well posed when $U_{1} U_{2} U_{1}^{\dagger} U_{2}^{\dagger}$ has no real negative eigenvalue. $\operatorname{Bott}\left(U_{1}, U_{2}\right)=0$ if and only if $U_{1}$ and $U_{2}$ are arbitrarily close to a couple of commuting quasiunitaries [20]. This has been shown to be in relation with the existence of exponentially localized Wannier functions. More precisely, the existence of exponentially localized Wannier functions implies the vanishing of the Bott index, while the vanishing of the Bott implies a spread of the Wannier functions, quantified by their variance, that is small compared with the linear size of the system [22,23]. An equivalent definition of the Bott index is given employing the matrices $V_{1} \equiv Q+P e^{\left(i \frac{2 \pi X}{L}\right)} P$ and $V_{2} \equiv Q+P e^{\left(i \frac{2 \pi Y}{L}\right)} P$, then

$$
\operatorname{Bott}\left(U_{1}, U_{2}\right)=\frac{1}{2 \pi} \operatorname{Im} \operatorname{Tr} \log \left(V_{1} V_{2} V_{1}^{\dagger} V_{2}^{\dagger}\right) \text {. }
$$
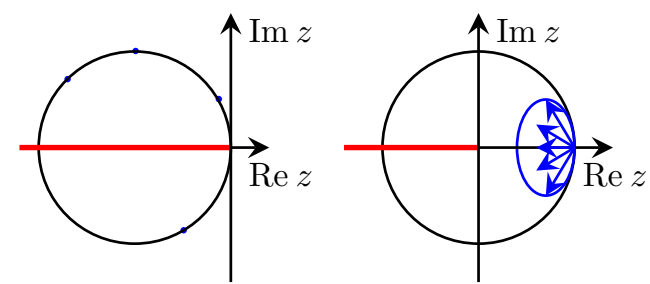

FIG. 1. Left panel: with $U_{1}$ and $U_{2}$ unitary the spectrum of $U_{1} U_{2} U_{1}^{\dagger} U_{2}^{\dagger}-1$ is a set of points on the black circle of radius equal to 1 . $\operatorname{det}\left(U_{1} U_{2} U_{1}^{\dagger} U_{2}^{\dagger}\right)=1$. The only point at a distance equal to 2 from the origin is on the real negative axis. The thick red line indicates the branch cut of the logarithm. Right panel: the spectrum of $V_{1} V_{2} V_{1}^{\dagger} V_{2}^{\dagger}$ as given in Eq. (18) is confined in a small region close to $(1,0)$. The unitary time evolution of the system is such that $P$ and $Q$ are replaced by $U\left(t, t_{0}\right) P\left(t_{0}\right) U^{\dagger}\left(t, t_{0}\right)$ and $U\left(t, t_{0}\right) Q\left(t_{0}\right) U^{\dagger}\left(t, t_{0}\right)$ as a consequence the eigenvalues of $V_{1}(t) V_{2}(t) V_{1}^{\dagger}(t) V_{2}^{\dagger}(t)$ can move in time but nevertheless they stay confined within the unit disk in an area close by $(1,0)$ schematically drawn as an ellipse. The thick red line indicates the branch cut of the logarithm.

The proof of the equivalence is immediate using the representation of the projections $P$ and $Q$ as in Eqs. (9) and (10).

With the use of conditions (15) and (16), it is possible to show that

$$
\begin{aligned}
\operatorname{Bott}\left(U_{1}, U_{2}\right)= & \frac{1}{2 \pi} \operatorname{Im} \operatorname{Tr}\left(P e^{i \theta_{x}} P e^{i \theta_{y}} P e^{-i \theta_{x}} P e^{-i \theta_{y}} P\right) \\
& +O\left(L^{-2}\right)
\end{aligned}
$$

with $\theta_{x} \equiv \frac{2 \pi X}{L}$ and $\theta_{y} \equiv \frac{2 \pi Y}{L}$. This expression is particularly well suited for numerical investigations.

Let us consider how the Bott index varies, starting with the simpler case of unitary matrices, namely, when $U_{1}$ and $U_{2}$ in Eq. (17) are replaced by unitaries. In this case, the Bott index is well defined when $\left\|\left[U_{1}, U_{2}\right]\right\|<2$ because with $U_{1}$ and $U_{2}$ unitary $U_{1} U_{2} U_{1}^{\dagger} U_{2}^{\dagger}$ is unitary as well; that means its spectrum lies on the unit circle of the complex plane. It is easy to see that $\left\|\left[U_{1}, U_{2}\right]\right\|=\left\|U_{1} U_{2} U_{1}^{\dagger} U_{2}^{\dagger}-1\right\|$, then recalling that the operator norm of a matrix $A$ is $\|A\| \equiv \max \{|\lambda|, \lambda \in \sigma(A)\}$ we see in the left panel of Fig. 1 that $\left\|U_{1} U_{2} U_{1}^{\dagger} U_{2}^{\dagger}-1\right\|=2$ if and only if -1 belongs to the spectrum of $U_{1} U_{2} U_{1}^{\dagger} U_{2}^{\dagger}$. Then, when an eigenvalue crosses the real negative axis, that corresponds to cross the branch cut of the logarithm, its phase changes of $2 \pi$ then the Bott index (17) changes. In Ref. [24] it is discussed how a deformation (a homotopy) of a couple of unitary matrices that preserves their unitarity can lead to a change of their Bott index only if at an intermediate point $\left\|\left[U_{1}, U_{2}\right]\right\|=$ 2. Namely, given a homotopy $t \rightarrow\left(U_{t}, V_{t}\right)$ with $\left(U_{t}, V_{t}\right)$ unitary matrices $\forall t \in[0,1]$ then $\operatorname{Bott}\left(U_{0}, V_{0}\right) \neq \operatorname{Bott}\left(U_{1}, V_{1}\right)$ only if it exists $\bar{t} \in[0,1]$ such that $\left\|\left[U_{\bar{t}}, V_{\bar{t}}\right]\right\|=2$. See [24] and references therein for more theorems about it. In the case of the definition (17) where $U_{1}$ and $U_{2}$ are not unitary, we must consider where the spectrum of $U_{1} U_{2} U_{1}^{\dagger} U_{2}^{\dagger}$ is located. It is possible to see (see Appendix for the details) that for a time-independent system the spectrum is located close by the point $(1,0)$ of the complex plane; this follows directly from Eqs. (15) and (16). When $U\left(t, t_{0}\right)$ commutes with $P\left(t_{0}\right)$, the spectrum stays in the same region. When instead $U\left(t, t_{0}\right)$ does not commute with $P\left(t_{0}\right)$, as explained in the next section, the 
time evolution causes the eigenvalues to move within a small subregion of the disk of unit radius (see the right panel of Fig. 1).

\section{INVARIANCE OF THE BOTT INDEX OF THE TIME-EVOLVED FERMI PROJECTION}

The Chern number is invariant under unitary evolution of a generic local and time-dependent Hamiltonian. We ask the same question about the Bott index. A way to realize the time driving of an initial Hamiltonian $H_{i}$ towards a final Hamiltonian $H_{f}(t)$ is the ramp of a perturbation $V(t)$ in general time-dependent:

$$
H_{f}(t)=H_{i}+r(t) V(t), \quad r(t)= \begin{cases}0, & t<t_{0} \\ v(t), & t_{0} \leqslant t \leqslant t_{1} \\ 1, & t>t_{1}\end{cases}
$$

with $v(t)$ a monotonic regular function interpolating between zero and one. When the slope of $v(t)$ increases significantly, the driving becomes a so-called quantum quench. In this case the operator of unitary evolution from the initial Hamiltonian $H_{i}$ to the final Hamiltonian $H_{f}=H_{i}+V$ is given in the case of $V$ time independent by

$$
U\left(t, t_{0}\right)=U\left(t, t_{1}\right) U\left(t_{1}, t_{0}\right)=e^{-i\left(t-t_{1}\right) H_{f}} U\left(t_{1}, t_{0}\right)
$$

with

$$
t_{1}-t_{0} \ll\left(\Delta_{\psi\left(t_{0}\right)} V\right)^{-1}
$$

the operator $U\left(t_{1}, t_{0}\right) \simeq \mathbb{1}$. In Eq. (22)

$$
\Delta_{\psi\left(t_{0}\right)} V \equiv \sqrt{\left\langle\psi\left(t_{0}\right)\left|V^{2}\right| \psi\left(t_{0}\right)\right\rangle-\left(\left\langle\psi\left(t_{0}\right)|V| \psi\left(t_{0}\right)\right\rangle\right)^{2}}
$$

is the variance of the perturbing potential over the initial state $\left|\psi\left(t_{0}\right)\right\rangle$. This is discussed for example in [39].

Let us study the time evolution of the Bott index. This can be done in the Schrödinger picture replacing $P\left(t_{0}\right)$ with $U\left(t, t_{0}\right) P\left(t_{0}\right) U^{\dagger}\left(t, t_{0}\right)$ and $Q\left(t_{0}\right)$ with $U\left(t, t_{0}\right) Q\left(t_{0}\right) U^{\dagger}\left(t, t_{0}\right)$. The invariance for a time-independent system is manifest, in fact $P\left(t_{0}\right)$ and $U\left(t, t_{0}\right)=e^{-i\left(t-t_{0}\right) H}$ commute. I stress that this is different from considering the instantaneous Bott index that we would get replacing $P$ with $P(t)=\sum_{i}\left|\psi_{i}(t)\right\rangle\left\langle\psi_{i}(t)\right|$ being $\left|\psi_{i}(t)\right\rangle$ the instantaneous eigenvector of the Hamiltonian $H(t)\left|\psi_{i}(t)\right\rangle=E_{i}(t)\left|\psi_{i}(t)\right\rangle, E_{i}(t) \leqslant \mu$. Is the invariance of the Bott index of the time-evolved Fermi projection also granted for a general time-dependent system with Hamiltonian (20)? A variation of the Bott index has been numerically shown in [5]; in the rest of this section I show that this can happen only as a finite-size effect. The Bott index does not change due to the unitary evolution generated by a local Hamiltonian in the thermodynamic limit.

The analysis of the unitary case showed that the variation of the Bott index is due to the growth of $\left\|\left[U_{1}, U_{2}\right]\right\|$. In our physical context, the matrices $U_{1}$ and $U_{2}$ are not unitary and the increase of the norm of their commutator with time is due to the growth of $\left\|\left[e^{i \theta_{x}}, U\left(t, t_{0}\right) H\left(t_{0}\right) U^{\dagger}\left(t, t_{0}\right)\right]\right\|=$ $\left\|\left[e^{i \theta_{x, H}(t)}, H\right]\right\|$ with $\theta_{x, H}(t) \equiv U^{\dagger}\left(t, t_{0}\right) \theta_{x} U\left(t, t_{0}\right)$ the Heisenberg picture of $\theta_{x}$. This determines in principle the growth of $\left\|\left[e^{i \theta_{x}}, U\left(t, t_{0}\right) P U^{\dagger}\left(t, t_{0}\right)\right]\right\|=\left\|\left[e^{i \theta_{x, H}(t)}, P\right]\right\|$ in time. Let us examine this explicitly.
A time-independent, short-ranged, bounded, and gapped Hamiltonian implies in a system large compared to the range $R$ the relations (13), (14), (15), and (16) above. In the general setting of a time-dependent Hamiltonian, that might be associated to the ramp of a time-dependent perturbation, the unitary operator of time evolution, also called the propagator, is $U\left(t, t_{0}\right)=T \exp \left(-i \int_{t_{0}}^{t} d s H(s)\right), T$ denotes the operator of time ordering. $U\left(t, t_{0}\right)$ does not commute in general with the Hamiltonian $H(t)$. A possible way to examine $\left\|\left[U^{\dagger}\left(t, t_{0}\right) X U\left(t, t_{0}\right), H\right]\right\|$ is to use the Lieb-Robinson bounds $[40,41]$, but these are better suited for operators that have supports that do not overlap, therefore, I employ a different strategy.

The equation of motion for $X_{H}(t) \equiv U^{\dagger}\left(t, t_{0}\right) X U\left(t, t_{0}\right)$ is $i \frac{d}{d t} X_{H}(t)=[X, H(t)]_{H}$. The explicit time dependence of the Hamiltonian has been put in evidence. With $X=X\left(t_{0}\right)$ we have

$$
i\left[X_{H}(t)-X\right]=\int_{t_{0}}^{t} d s[X, H(s)]_{H} .
$$

Being $\left\|\left[X, U\left(t, t_{0}\right)\right]\right\|=\left\|U^{\dagger}\left(t, t_{0}\right) X U\left(t, t_{0}\right)-X\right\|$ it follows that

$$
\left\|\left[X, U\left(t, t_{0}\right)\right]\right\| \leqslant\left|t-t_{0}\right| \sup _{s \in\left[t_{0}, t\right]}\|[X, H(s)]\|
$$

using Eq. (13) and denoting $R(t)$ the range of $H(t)$ and $J(t)$ its norm, it follows that

$$
\left\|\left[X, U\left(t, t_{0}\right)\right]\right\| \leqslant\left|t-t_{0}\right| \sup _{s \in\left[t_{0}, t\right]} 2 R(s) J(s) .
$$

Our interest is in the growth with time of $\left\|\left[U^{\dagger}\left(t, t_{0}\right) X U\left(t, t_{0}\right), H\left(t_{0}\right)\right]\right\|$. It follows from Eqs. (13) and (24), we drop the time indices of $U\left(t, t_{0}\right)$ and set $J \equiv J\left(t_{0}\right)$, that

$$
\begin{aligned}
\left\|\left[U^{\dagger} X U, H\left(t_{0}\right)\right]\right\| & =\left\|\left[U^{\dagger}[X, U]+X, H\left(t_{0}\right)\right]\right\| \\
& =\left\|\left[U^{\dagger}[X, U], H\left(t_{0}\right)\right]+\left[X, H\left(t_{0}\right)\right]\right\| \\
& \leqslant\left\|\left[U^{\dagger}[X, U], H\left(t_{0}\right)\right]\right\|+2 R J \\
& \leqslant 2 J\|[X, U]\|+2 R J \\
& \leqslant 4 J\left|t-t_{0}\right| \sup _{s \in\left[t_{0}, t\right]}(R(s) J(s))+2 R J .
\end{aligned}
$$

In analogy to the static case, where Eq. (13) implied Eq. (14), in the time-dependent case we have, being $P \equiv P\left(t_{0}\right)$ the Fermi projection,

$$
\frac{\left\|\left[U^{\dagger} X U, P\right]\right\|}{L} \leqslant \frac{4\left|t-t_{0}\right| \sup _{s \in\left[t_{0}, t\right]}(R(s) J(s))}{L}+\frac{2 R J}{L \Delta E} .
$$

A necessary condition for the change of the Bott index is

$$
\frac{\left\|\left[U^{\dagger} X U, P\right]\right\|}{L} \simeq 1 \text {. }
$$

This corresponds to a lower bound for the time interval $\bar{t}-t_{0}$ that would give rise to a change of the index such that

$$
\bar{t}-t_{0} \gtrsim \frac{L-\frac{2 R J}{\Delta E}}{4 \sup _{s \in\left[t_{0}, \bar{t}\right]}(R(s) J(s))} .
$$

For a finite-range Hamiltonian in the thermodynamic limit $\frac{L}{R} \rightarrow \infty$, therefore, if the instantaneous Hamiltonian $H(t)$ has 
always a finite range $R(t)$, the timescale for the variation of the index diverges. In a finite-size setting, the interplay of the ratios $\frac{L}{R} \gg 1$ and $\frac{J}{\Delta E} \gg 1$ might lead to an experimentally meaningful value of the timescale given in Eq. (27), that nevertheless corresponds to a lower bound, for the change of the Bott index; for this reason the present estimate does not dismiss the numerical results of [5].

\section{Periodically driven systems}

Let us consider the case in Eq. (20) of the ramp of a timeperiodic perturbation $V(t)=V(t+n T), \forall n \in \mathbb{Z}$. This implies that the Hamiltonian $H(t)$ when $t \geqslant t_{1}$ is time periodic. In this case, the estimate given by Eq. (24) can be made sharper. In general, the propagator $U$ of a time-periodic Hamiltonian is not periodic, but when $U$ has a spectral gap then there is a homotopy that maps $U$ to a time-periodic propagator preserving the given gap as described in Ref. [10]. The Hamiltonian that generates this periodic propagator is called the relative Hamiltonian, and its construction is described, for example, in [10-12]. The propagator $U$ and the homotopically equivalent time-periodic propagator share the same topological index $W^{\varepsilon}$ of [10] (see [11] for a different naming), that characterizes each spectral gap of the propagator placed at $e^{-i T \varepsilon}$ and therefore the Chern number of the spectral projection in-between the various gaps. In fact, denoting $P^{\varepsilon, \varepsilon^{\prime}}$ the spectral projection for the spectrum of $U$ in-between the gaps $e^{-i T \varepsilon}$ and $e^{-i T \varepsilon^{\prime}}$ it holds $W^{\varepsilon}-W^{\varepsilon^{\prime}}=\operatorname{Chern}\left(P^{\varepsilon^{\prime}, \varepsilon}\right)$. This is Eq. 14 of [10] or Eq. 3.22 of [11].

The propagator $U$ of a time-periodic Hamiltonian of period $T$ is such that $\forall n \in \mathbb{Z}: U\left(t+n T, t_{1}+n T\right)=$ $U\left(t, t_{1}\right)$. The supposed periodicity of $U$ means that $U\left(t_{1}+\right.$ $\left.n T, t_{1}\right)=\mathbb{1}, \forall n \in \mathbb{Z}$. Therefore, given $t$ and $t_{1}$, since it exists a positive integer $N$ such that $t-\left(t_{1}+N T\right)<$ $T$, we have that $U\left(t, t_{1}\right)=U\left(t, t_{1}+N T\right) U\left(t_{1}+N T, t_{1}\right)=$ $U\left(t, t_{1}+N T\right)$. We suppose that according to Eq. (20) the time needed to fully turn on the time-periodic perturbation $V(t)=V(t+n T), \forall n \in \mathbb{Z}$ is equal to $t_{1}-t_{0}$, then the propagator of the Hamiltonian of Eq. (20) for a suitable $N$, denoting $U_{\text {per }}$ the periodic propagator, obeys the decomposition

$$
\begin{aligned}
U\left(t, t_{0}\right) & =U_{\mathrm{per}}\left(t, t_{1}\right) U_{\mathrm{ramp}}\left(t_{1}, t_{0}\right) \\
& =U_{\mathrm{per}}\left(t, t_{1}+N T\right) U_{\mathrm{ramp}}\left(t_{1}, t_{0}\right) \\
& =U_{\mathrm{per}}\left(t-N T, t_{1}\right) U_{\mathrm{ramp}}\left(t_{1}, t_{0}\right) .
\end{aligned}
$$

In this way we conclude that the time interval $\left|t-t_{0}\right|$ of Eq. (25) is less than $t_{1}+T-t_{0}$. Therefore, for a periodic driving the change of the Bott index is not only forbidden at any given fixed $\left|t-t_{0}\right|$ in the thermodynamic limit, but also disfavored with respect to the general case for a finite-size setting.

I stress that the relative Hamiltonian of a space-local Hamiltonian under the hypothesis of existence of a spectral gap for the propagator is also space local. This has been shown in proposition 5.6 of Ref. [11] (see also the specific notion of locality employed in that reference).

This discussion ignores possible heating effects that might take place in the context of periodic driving, nevertheless, recent works indicate the stability of such a phases over almost exponentially long times [42-44].

\section{CONCLUDING DISCUSSION}

The Bott index introduced in the physics realm in Refs. [20,22,23] has been investigated in a general [Eq. (20)] time-dependent setting and the constancy of the index of the time-evolved Fermi projection has been established in the thermodynamic limit. The timescale of a possible change of the index is identified in Eq. (25). This is a mere finite-size effect, in particular, it looks disfavored in the timeperiodic case.

A fundamental issue unexplored in this work is the meaning as a physical quantity of the Bott index for a general time-dependent Hamiltonian. In fact, if for a static system it is equivalent to the Chern number so it measures the Hall conductance, what about instead a general time-dependent system? We should recall that the Hall conductance is not the mean value of an operator over a state but a transport coefficient computed with the aid of the Kubo formula. The Hall conductance has been found to be not quantized after a quench according to Refs. [7-9]. The physical meaning of the quantized Bott index for a general time-dependent setting remains to be investigated.

I conclude with some final comments about the literature. In Ref. [45] the Bott index has been claimed to be the right suited invariant to study finite systems that are disordered and periodically driven being the counterpart of the winding number invariant $W$ introduced in the Ref. [10] for the study of clean and thermodynamically large periodically driven systems. W counts the number of edge states supported by a periodically driven two-dimensional system. Let me comment briefly on the relation among Refs. [5,10]: to me, one of the most interesting results of [5] is to show that after ramping up a circularly polarized electric field on a graphene sheet with a staggering sublattice potential, the initial ground state evolves keeping a vanishing Chern number despite the fact that the ground state of the final periodic Hamiltonian has a nontrivial Chern number. This was shown in Sec. II using the invariance of the Chern number of homotopically equivalent projections and discussing the trace class properties of the operator on the right-hand side of Eq. (6). Reference [10], on the other hand, considers a system that is already in a periodically driven regime disregarding the effects of the ignition of the drive. The issues related to the preparation of a periodically driven system are also discussed, e.g., in Ref. [46]. Finally, it needs to be mentioned that all the effects associated with phonons and their coupling with electrons have been neglected. For a study that takes these phenomena into account in the context of a quench of topological phases, see [47].

\section{ACKNOWLEDGMENTS}

It is a pleasure to thank Y. Ge and M. Rigol for exchange of correspondence, and H. Schulz-Baldes, Y. Avron, J. Shapiro, and F. Dorsch for discussions. 


\section{APPENDIX}

We want to estimate the eigenvalues with maximum modulus (that is the norm) and the minimum modulus of the matrix $Q+P e^{i \theta_{x, H}} P e^{i \theta_{y, H}} P e^{-i \theta_{x, H}} P e^{-i \theta_{y, H}} P$ that is the argument of the logarithm that defines the Bott index (18). The subscript $H$ indicates the Heisenberg picture. For simplicity, we start considering $Q+P e^{i \theta_{x, H}} P$. This matrix is not normal, but it admits a singular value decomposition: $Q+P e^{i \theta_{x, H}} P=T^{\dagger} D S$, $T$ and $S$ are unitary, $D$ is the diagonal matrix of eigenvalues. Then, the modulus square of the eigenvalues of $Q+P e^{i \theta_{x, H}} P$ are the eigenvalues of $G \equiv Q+P e^{-i \theta_{x, H}} P e^{i \theta_{x, H}} P$. In fact,

$$
\begin{gathered}
G=\left(Q+P e^{i \theta_{x, H}} P\right)^{\dagger}\left(Q+P e^{i \theta_{x, H}} P\right) \\
=S^{\dagger} D^{\dagger} T T^{\dagger} D S=S^{\dagger} D^{\dagger} D S .
\end{gathered}
$$

In the energy basis

$$
P=\left(\begin{array}{cc}
0 & 0 \\
0 & \mathbb{1}_{n}
\end{array}\right), \quad Q=\left(\begin{array}{cc}
\mathbb{1}_{m} & 0 \\
0 & 0
\end{array}\right) .
$$

Then, it is immediate to see that

$$
G=\left(\begin{array}{cc}
\mathbb{1}_{m} & 0 \\
0 & W_{4}^{\dagger} W_{4}
\end{array}\right)
$$

with $W_{4}$ the lower diagonal block of the unitary matrix

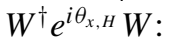

$$
W^{\dagger} e^{i \theta_{x, H}} W=\left(\begin{array}{ll}
W_{1} & W_{2} \\
W_{3} & W_{4}
\end{array}\right) .
$$

From Eq. (A4) we get

$$
\|G\|=\max \left\{1,\left\|W_{4}^{\dagger} W_{4}\right\|\right\}=1 .
$$

Note that $W_{4}$ satisfies, for example, $W_{2}^{\dagger} W_{2}+W_{4}^{\dagger} W_{4}=\mathbb{1}$, then being $W_{2}^{\dagger} W_{2}$ semipositive definite we have $\left\|W_{4}\right\| \leqslant 1$. $W_{2}$ can be vanishing only when $W$ commutes with $e^{-i \theta_{x, H}}$, and moreover when $U\left(t, t_{0}\right)$ commutes with $H\left(t_{0}\right)$ that is not the case we are interested in here. Then, it follows that

$$
\left\|Q+P e^{i \theta_{x, H}} P\right\|=1 \text {. }
$$

With a similar argument we also obtain that

$$
\left\|Q+P e^{i \theta_{x, H}} P e^{i \theta_{y, H}} P e^{-i \theta_{x, H}} P e^{-i \theta_{y, H}} P\right\|=1
$$

Let us investigate the eigenvalue of minimum modulus of $Q+$ $P e^{i \theta_{x, H}} P e^{i \theta_{y, H}} P e^{-i \theta_{x, H}} P e^{-i \theta_{y, H}} P$. We again start considering the matrix $Q+P e^{i \theta_{x, H}} P$ for simplicity. Its eigenvalue of minimum modulus is the square root of the smallest eigenvalue of $G$ that I indicate with $\lambda_{S}$. It is easy to see using the positivity of $G$ and $\|G\|=1$ that

$$
\begin{gathered}
1-\lambda_{S}=\|\mathbb{1}-G\|=\left\|\mathbb{1}-Q-P e^{-i \theta_{x, H}} P e^{i \theta_{x, H}} P\right\| \\
=\left\|P\left(\mathbb{1}-e^{-i \theta_{x, H}} P e^{i \theta_{x, H}}\right) P\right\| \\
=\left\|P e^{-i \theta_{x, H}} Q e^{i \theta_{x, H}} P\right\| \\
=\left\|\left[P, e^{-i \theta_{x, H}}\right] Q\left[e^{i \theta_{x, H}}, P\right]\right\|
\end{gathered}
$$

Equation (A12) is a clear indication that when $\left[e^{i \theta_{x, H}}, P\right]$ is small, then $\lambda_{S}$ is close to 1 . This follows directly from Eq. (14) that in turn follows from the hypothesis on the Hamiltonian's properties: short ranged, bounded, gapped. We have seen that a result of the time evolution is to make the norm $\left\|\left[e^{i \theta_{x, H}}, P\right]\right\|$ growing, and this determines the decrease of $\lambda_{S}$. We guess that if $\left\|\left[e^{i \theta_{x, H}}, P\right]\right\| \simeq 1$, then $\lambda_{S}$ is small. We have seen that this is forbidden in the thermodynamic limit $L \rightarrow \infty$. A similar analysis brings to the same conclusions for the eigenvalue of minimum modulus of $Q+P e^{i \theta_{x, H}} P e^{i \theta_{y, H}} P e^{-i \theta_{x, H}} P e^{-i \theta_{y, H}} P$. As explained in the main text, the eigenvalues of this operator are at the beginning all close to the point $(1,0)$ of the complex plane. An equilibrium evolution would let them stay in that region in such a way that the Bott index does not change. The time evolution generated by a time-dependent Hamiltonian makes them move within the disk of unit radius but nevertheless in the thermodynamic limit they do not cross the real negative axis in such a way the Bott index is invariant along the time-unitary evolution of the system.
[1] N. H. Lindner, G. Refael, and V. Galitski, Nat. Phys. 7, 490 (2011).

[2] T. Oka and H. Aoki, Phys. Rev. B 79, 081406 (2009).

[3] T. Kitagawa, T. Oka, A. Brataas, L. Fu, and E. Demler, Phys. Rev. B 84, 235108 (2011).

[4] M. S. Foster, M. Dzero, V. Gurarie, and E. A. Yuzbashyan, Phys. Rev. B 88, 104511 (2013).

[5] L. D'Alessio and M. Rigol, Nat. Commun. 6, 8336 (2015).

[6] M. D. Caio, N. R. Cooper, and M. J. Bhaseen, Phys. Rev. Lett. 115, 236403 (2015).

[7] H. Dehghani, T. Oka, and A. Mitra, Phys. Rev. B 91, 155422 (2015).

[8] M. D. Caio, N. R. Cooper, and M. J. Bhaseen, Phys. Rev. B 94, 155104 (2016).

[9] P. Wang, M. Schmitt, and S. Kehrein, Phys. Rev. B 93, 085134 (2016)
[10] M. S. Rudner, N. H. Lindner, E. Berg, and M. Levin, Phys. Rev. X 3, 031005 (2013).

[11] G. M. Graf and C. Tauber, Ann. Henri Poincaré 19, 709 (2018).

[12] C. Sadel and H. Schulz-Baldes, Math. Phys. Anal. Geom. 20, 22 (2017).

[13] M. Fruchart, Phys. Rev. B 93, 115429 (2016).

[14] D. Carpentier, P. Delplace, M. Fruchart, and K. Gawedzki, Phys. Rev. Lett. 114, 106806 (2015).

[15] M. Tarnowski et al., arXiv:1709.01046 [cond-mat.quant-gas].

[16] M. B. Hastings and X.-G. Wen, Phys. Rev. B 72, 045141 (2005).

[17] S. Bachmann, W. De Roeck, and M. Fraas, Phys. Rev. Lett. 119, 060201 (2017).

[18] S. Bachmann, W. De Roeck, and M. Fraas, Commun. Math. Phys. 361, 997 (2018).

[19] J. C. Budich and M. Heyl, Phys. Rev. B 93, 085416 (2016). 
[20] T. A. Loring and M. B. Hastings, Europhys. Lett. 92, 67004 (2010).

[21] T. A. Loring, Can. J. Math. 40, 197 (1988).

[22] M. B. Hastings and T. A. Loring, J. Math. Phys. 51, 015214 (2010).

[23] M. B. Hastings and T. A. Loring, Ann. Phys. 326, 1699 (2011).

[24] T. A. Loring, Sigma 10, 077 (2014).

[25] D. Monaco, G. Panati, A. Pisante, and S. Teufel, arXiv:1612.09557 [cond-mat].

[26] Y. Ge and M. Rigol, Phys. Rev. A 96, 023610 (2017).

[27] D. Toniolo, arXiv:1708.05912 [cond-mat].

[28] S. Bravyi, M. B. Hastings, and F. Verstraete, Phys. Rev. Lett. 97, 050401 (2006).

[29] J. Bellissard, A. van Elst, and H. Schulz-Baldes, J. Math. Phys. 35, 5373 (1994).

[30] A. Kitaev, Ann. Phys. 321, 2 (2006).

[31] E. Prodan, Phys. Rev. B 80, 125327 (2009).

[32] E. Prodan, T. L. Hughes, and B. A. Bernevig, Phys. Rev. Lett. 105, 115501 (2010).

[33] E. Prodan, J. Phys. A: Math. Theor. 44, 113001 (2011).

[34] G. De Nittis, M. Drabkin, and H. Schulz-Baldes, Markov Processes and Related Fields 21, 463 (2015).
[35] A. Elgart, G. Graf, and J. Schenker, Commun. Math. Phys. 259, 185 (2005).

[36] J. Avron, R. Seiler, and B. Simon, Commun. Math. Phys. 159, 399 (1994).

[37] B. Simon, Operator Theory: A Comprehensive Course in Analysis, Part 4 (AMS, Providence, RI, 2015).

[38] M. Reed and B. Simon, Methods of Modern Mathematical Physics, Vol. 2 (Academic, New York, 1975).

[39] A. Galindo and R. Pascual, Quantum Mechanics II (Springer, Berlin, 1991).

[40] E. H. Lieb and D. W. Robinson, Commun. Math. Phys. 28, 251 (1972).

[41] M. B. Hastings, arXiv:1008.5137 [math-ph].

[42] A. Russomanno, A. Silva, and G. E. Santoro, Phys. Rev. Lett. 109, 257201 (2012).

[43] D. A. Abanin, W. De Roeck, and F. Huveneers, Phys. Rev. Lett. 115, 256803 (2015).

[44] D. Abanin, W. De Roeck, W. W. Ho, and F. Huveneers, Commun. Math. Phys. 354, 809 (2017).

[45] P. Titum, N. H. Lindner, M. C. Rechtsman, and G. Refael, Phys. Rev. Lett. 114, 056801 (2015).

[46] W. W. Ho and D. A. Abanin, arXiv:1611.05024 [cond-mat].

[47] M. Schüler and P. Werner, Phys. Rev. B 96, 155122 (2017). 